\title{
Dynamics and Shape of Brightest Cluster Galaxies
}

\author{
H. Andernach ${ }^{1}$, K. Alamo-Martínez ${ }^{1}$, R. Coziol $^{1}$ and E. Tago ${ }^{2}$ \\ 1 Departamento de Astronomía, Universidad de Guanajuato, Mexico \\ heinz@astro.ugto.mx, karla@astro.ugto.mx, rcoziol@astro.ugto.mx \\ 2 Tartu Observatory, Tõravere, Estonia erik@aai.ee
}

Summary. We identified Brightest Cluster Members (BCM) on DSS images of 1083 Abell clusters, derived their individual and host cluster redshifts from literature and determined the BCM ellipticity. Half the BCMs move at a speed higher than $37 \%$ of the cluster velocity dispersion $\sigma_{\mathrm{cl}}$, suggesting that most BCMs are part of substructures falling into the main cluster. Both, the BCM's velocity offset in units of $\sigma_{\mathrm{cl}}$, and BCM ellipticity, weakly decrease with cluster richness.

If BCMs formed "in-situ" they should "rest" at the bottom of the potential well of the cluster and have a radial velocity equal to the cluster mean. Previous studies, e.g. [4, based on small samples of clusters dominated by a $\mathrm{cD}$ galaxy, showed that this is far from reality. Here we extend these studies to 1083 Abell clusters likely to have a dominant galaxy, namely all clusters with Bautz-Morgan (BM) types I or I-II, all with Rood-Sastry (RS) type $\mathrm{cD}$, and all clusters with notes in [1 indicating a "corona" of the first-ranked galaxy. From DSS images we derived positions for 1329 BCM candidates in 1076 clusters (occasionally more than one candidate per cluster), retrieved their basic parameters from NED, and extracted cluster mean redshifts, $\mathrm{z}_{\mathrm{cl}}$, and velocity dispersions, $\sigma_{\mathrm{cl}}$, from the compilation maintained by two of us 2. Deleting all foreground/background BCM candidates and restriction to clusters with $\geq 10$ measured redshifts, yielded a sample of 385 BCMs in 326 Abell clusters for which we derived the relative velocity offset, $\mathrm{v}_{\text {off }} / \sigma_{\mathrm{cl}}=\left(\mathrm{v}_{\mathrm{BCM}}-\mathrm{cz}_{\mathrm{cl}}\right) /\left(1+\mathrm{z}_{\mathrm{cl}}\right) / \sigma_{\mathrm{cl}}$, with $\mathrm{v}_{\mathrm{BCM}}$ the BCM radial velocity.

In Fig. \we show that half of the BCMs in our sample move at peculiar velocities $>0.37 \sigma_{\mathrm{cl}}$. There is a trend for a smaller $\mathrm{v}_{\mathrm{off}} / \sigma_{\mathrm{cl}}$ in richer clusters which is expected if the latter are dynamically more evolved.

We also determined BCM ellipticities using IRAF's ellipse for 1193 BCM candidates in 1012 clusters, but restricted our statistical analysis to the same sample of 385 BCMs mentioned above. Figure 2 shows that BCM ellipticity increases with richness. This may suggest that BCMs in richer clusters grow more likely by anisotropic mergers.

Our observations are consistent with a model where most BCM form during the collapse and virialization of poor clusters or compact groups with low velocity dispersions 3 . This supports the view that most galaxies formed in groups (and not in rich clusters) with a common dark halo and/or individual halo of each galaxy which form(s) a local potential minimum for the BCM. 

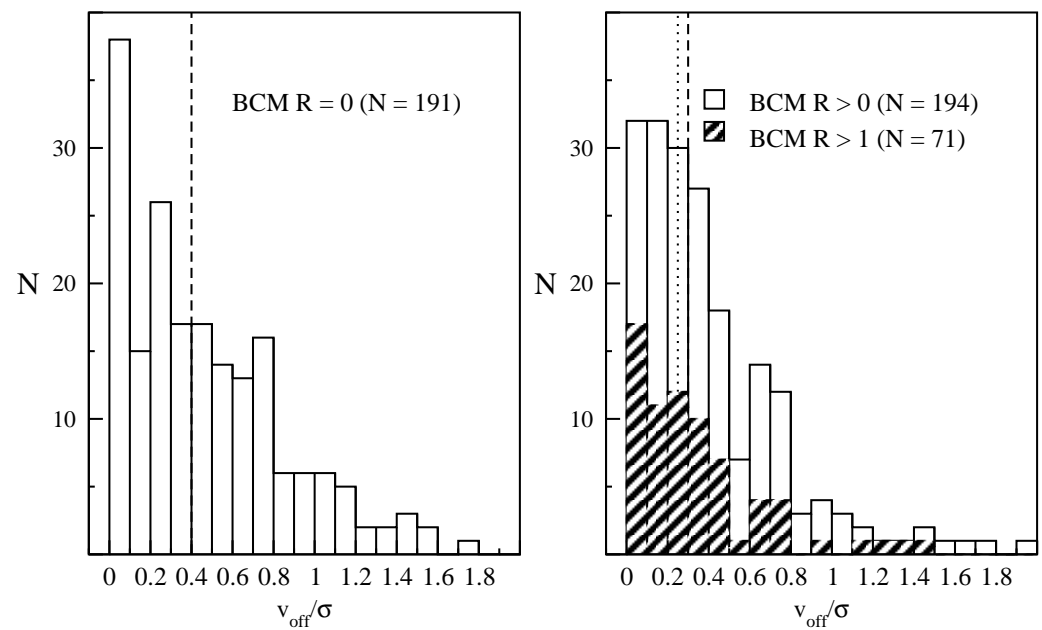

Fig. 1. Relative velocity offset for clusters with different Abell richness R. Median values are indicated with dashed lines or a dotted line for the shaded histogram.
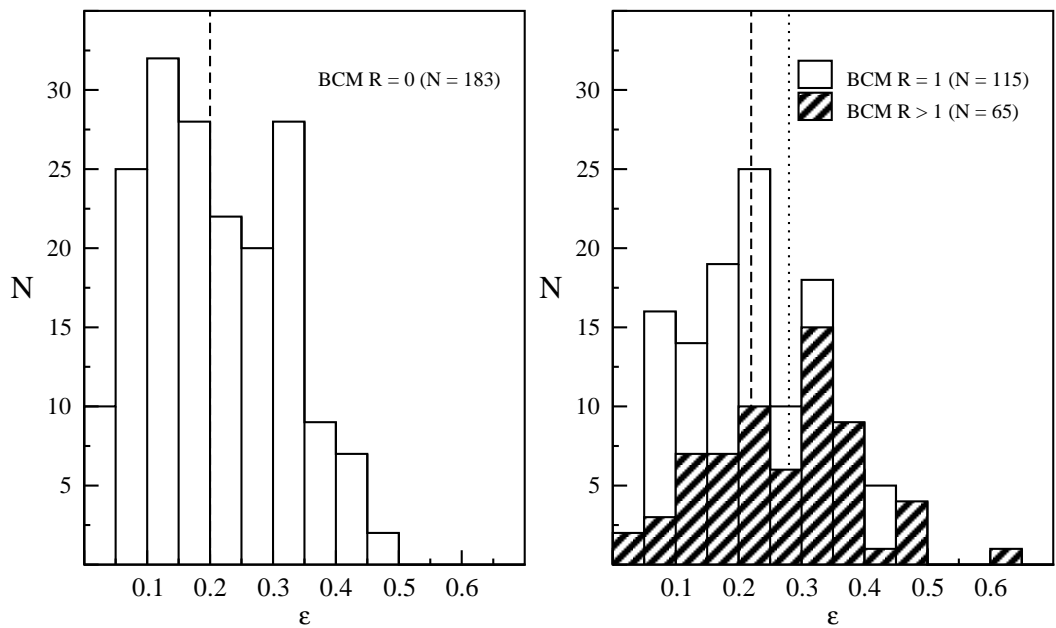

Fig. 2. BCM ellipticity distribution in clusters of different Abell richness. Median values are indicated with dashed lines or a dotted line for the shaded histogram.

\section{References}

1. Abell G.O., Corwin Jr. H.G., Olowin R.P., ApJS 70, 1 (1989)

2. Andernach H., Tago E., Einasto M., Einasto J., Jaaniste J.: ASP Conference Series, Vol. 239, eds. A.P. Fairall \& P.A. Woudt, p. 283 (2005)

3. Merritt D.: ApJ, 289, 18 (1985)

4. Zabludoff A.I., Geller M.J., Huchra J.P., Vogeley, M.S.: AJ, 106, 1273 (1993) 